\title{
Double jeu
}

DOUBLE JEU Théâtre / Cinéma

\section{De Rachel à Sarah Bernhardt ou la naissance de l'antisémitisme culturel}

\section{Chantal Meyer-Plantureux}

\section{(2) OpenEdition}

1 Journals

Édition électronique

URL : http://journals.openedition.org/doublejeu/1505

DOI : 10.4000/doublejeu.1505

ISSN : 2610-072X

Éditeur

Presses universitaires de Caen

\section{Édition imprimée}

Date de publication : 1 avril 2009

Pagination : 15-36

ISBN : 978-2-84133-320-2

ISSN : $1762-0597$

\section{Référence électronique}

Chantal Meyer-Plantureux, « De Rachel à Sarah Bernhardt ou la naissance de l'antisémitisme

culturel », Double jeu [En ligne], 5 | 2009, mis en ligne le 05 juillet 2018, consulté le 20 novembre 2020

URL : http://journals.openedition.org/doublejeu/1505; DOI : https://doi.org/10.4000/doublejeu.1505

\section{(c) (7) (8)}

Double Jeu est mise à disposition selon les termes de la Licence Creative Commons Attribution - Pas d'Utilisation Commerciale 4.0 International. 


\title{
DE RACHEL À SARAH BERNHARDT OU LA NAISSANCE DE L'ANTISÉMITISME CULTUREL
}

\author{
LE ROMANTISME \\ ou le « qÉNIE CRÉAteUr des juifs »
}

C'est par l'instruction et la culture que s'effectuera la véritable intégration ${ }^{1}$ des juifs dans la société française. Comme Alfred de Vigny le fait remarquer avec une certaine ambiguïté, la deuxième génération (après l'émancipation de 1791) qui a bénéficié de l'instruction publique va brillamment réussir:

Cette race orientale et enflammée, race directe des patriarches, remplie de toutes les lumières et de toutes les harmonies primitives, a des aptitudes supérieures qui la mènent au sommet de tout, dans les affaires, les lettres et surtout les arts et la musique avant les beaux-arts.

Il est vrai que «la musique et le théâtre, secteurs moins conventionnels et moins conformistes ont très tôt accueilli des juifs $»^{2}$. Les cantatrices Giuditta Pasta (1797-1865) et Cornélie Falcon (1814-1897) qui créa le personnage de

1. Le 27 septembre 1791 parait le décret d'émancipation de tous les juifs de France; ils seront les premiers en Europe à être émancipés mais la Terreur en 1793 avec sa politique antireligieuse n'épargnera pas les juifs. C'est ensuite Bonaparte qui proclamera la liberté des cultes quand Napoléon en 1808 avec ce qu'on a appelé «le décret infâme» remettra les juifs hors du droit commun. Louis XVIII ne prorogera pas ces lois discriminatoires - elles auront duré 10 ans - et la première moitié du XIX ${ }^{e}$ siècle verra l'intégration progressive des juifs dans la nation française. À partir de 1846, plus une seule loi écrite ne distingue les juifs des autres citoyens français.

2. Esther Benbassa, Histoire des juifs de France, Paris, Seuil (Points Histoire), 2000. 
Rachel dans La Juive, les compositeurs Meyerbeer (1791-1864) et Halévy (1799-1862), première génération d'artistes musiciens juifs, ne se virent pas contester leur talent. Comme Rachel au théâtre. Mais ils étaient encore très peu nombreux à embrasser des carrières artistiques, ce qui peut expliquer une relative tolérance à leur égard.

Les juifs connaissent donc les premiers succès fulgurants dans le domaine de la musique: "La musique est avant tout un langage universel, dépassant les clivages géographiques, culturels ou religieux ${ }^{3}$. Fromenthal Halévy, Giacomo Meyerbeer ou Jacques Offenbach (1819-1880) un peu plus tard, seront reconnus et acclamés sans qu'ils n'aient à souffrir de véritable manifestation d'antisémitisme. Wagner, dont on verra plus tard les dérives antisémites admire profondément Meyerbeer. Depuis 1837, il a noué des relations avec lui: Wagner a fait de Meyerbeer son «dieu artistique, allemand et universel». La première lettre de Wagner adressée au musicien l'atteste: «Ce n'est pas l'endroit ici de multiplier les louanges maladroites de votre génie ${ }^{4}$. Le mot génie revient à plusieurs reprises dans les écrits de Wagner pour qualifier le musicien Meyerbeer. Meyerbeer l'aidera d'ailleurs par un grand nombre de lettres de recommandations lorsque Wagner viendra à Paris en 1839 et lui prêtera de l'argent. Fin 1840, Wagner écrit à Schumann pour lui demander de «ne pas laisse[r] éreinter Meyerbeer, je lui dois tout, et en particulier ma très prochaine célébrité ${ }^{5}$. Et dans une lettre de février 1842, il écrit à Meyerbeer: "L'éternité durant, je ne pourrai vous dire rien d'autre que merci, merci » ${ }^{6}$. Quant à Halévy, le jeune Wagner l'appréciera tant qu'il lui consacrera pas moins de quatre articles en 1842, regroupés ensuite en deux essais, Halévy et l'opéra français et La Reine de Chypre de Halévy; il parle d' "une musique qui jaillit des plus intimes et puissantes profondeurs de la nature humaine ${ }^{7}$. Il rencontre Halévy et s'en félicite:

Les arrangements de l'opéra d'Halévy me mirent en relation avec le maître lui-même et me procurèrent l'occasion de m'entretenir fréquemment et d'agréable manière avec cet homme excellent, vraiment modeste ${ }^{8} \ldots$

Et il précise :

3. Esther Benbassa, Histoire des juifs de France.

4. Léon Poliakov, Histoire de l'antisémitisme, Paris, Seuil (Points Histoire), 1991, t. 2, L’Âge de la science.

5. Ibid.

6. Ibid.

7. Cité par Frans C. Lemaire, Le Destin juif et la musique, Paris, Fayard, 2001.

8. Cité par Jean-Alexandre Ménétrier, «L'Amour triste: Fromenthal Halévy et son temps», L'Avant-Scène Opéra, n 100, juillet 1987, Fromenthal Halévy, La Juive. 
J'appréciais son talent vigoureux et je l'aimais pour son opéra de $L a$ Juive ${ }^{9}$.

La Révolution de 1848 scelle les noces des juifs et de la France: beaucoup affirmèrent leurs convictions républicaines. «Adolphe Crémieux et Michel Goudchaux sont nommés respectivement ministre de la Justice et ministre des Finances ${ }^{10}$. Et l'actrice Rachel (1821-1858) qui revendique ses origines juives et doit son nom à l'héroïne de l'opéra d'Halévy, $L a$ Juive - elle s'appelle en réalité Élisa - va devenir le symbole de la République française sur la scène de la Comédie française: drapée dans le drapeau français, elle déclame la Marseillaise ${ }^{11}$ (fig. 1) provoquant l'enthousiasme de Théophile Gautier:

Ce n'est pas là une vaine récitation [...] plus ou moins savamment modulée par les lèvres, c'est la poitrine qui éclate, la voix qui se brise, les nerfs qui tressaillent, les fibres qui palpitent; c'est une année de vie dépensée en dix minutes; c'est la haine, la fureur, la révolte, l'aspiration à la liberté, le patriotisme, les plus dévorantes passions humaines concentrées dans un frêle corps de jeune femme. [...] Avec quelques strophes, moitié chantées, moitié parlées, elle a fait tout un drame plein de grandeur et de passion, où frémissait l'âme d'un peuple s'éveillant à la liberté ${ }^{12}$...

L'extraordinaire popularité de la comédienne juive Rachel ne peut se comprendre que par l'analyse des conceptions esthétiques et politiques de l'époque. L'ascension fulgurante de l'actrice correspond à l'essor du romantisme, période caractérisée par la croyance en un destin personnel qui transcende à la fois les origines et les classes sociales: bien plus, le sort tragique du peuple juif et la misère de la famille Félix contribuent, dans l'esprit des romantiques, à en faire une figure d'exception.

C'est Victor Hugo qui proclame «l'indépendance du génie » : le romantisme cherche au plus profond du cœur ce que l'on a en soi de singulier. Alfred de Musset consacrera de nombreux textes au génie de Rachel:

[...] Une jeune fille qui n'a pas dix-sept ans, et qui semble n'avoir eu pour maître que la nature [...] Et où a-t-elle appris le secret d'une émotion

9. Ibid.

10. Esther Benbassa, Histoire des juifs de France.

11. En 1880, le jour anniversaire de la prise de la Bastille (14 juillet 1789) et celui de la fête de la Fédération (14 juillet 1790) deviennent fête nationale. Sarah Bernhardt prêtera aussi son concours à l'exécution de l'hymne national sur scène.

12. Théophile Gautier cité par Sylvie Vieilledent, «Gautier critique de Rachel», Bulletin de la Société Théophile Gautier, n²6, 2004, Théophile Gautier et le théâtre. C’est nous qui soulignons. 
si forte et si juste? Ni leçons, ni conseils, ni études, ne peuvent rien produire de semblable. [...] Il faut nécessairement reconnaître là une faculté divinatrice, inexplicable, qui trompe tous les calculs, et qui ressemble à ce qu'on appelle une révélation. Tel est le caractère du génie; il ne faut pas craindre de prononcer ce mot, car il est juste. Melle Rachel n'a pas un talent consommé, il s'en faut même de beaucoup, et cela lui reste à acquérir; elle a besoin d'étudier; mais on peut affirmer qu'elle a du génie, c'est-à-dire l'instinct du beau, du vrai, l'étincelle sacrée qui ne s'acquiert pas et qui ne se perd pas, quoi qu'on en dise ${ }^{13}$.

Théophile Gautier, ardent militant du romantisme ${ }^{14}$ et pourfendeur de la tragédie classique, sacre Rachel, actrice romantique: "La tragédie est le cadavre auquel Rachel infuse un sang neuf ${ }^{15}$. Gautier, dans un article de 1849, s'exclame:

Chose singulière! Mademoiselle Rachel, qui semblait avoir la terreur ou l'aversion de la poésie de son temps, ne s'apercevait pas qu'elle réussissait dans la tragédie par le sentiment tout moderne qu'elle y apportait. Le jeune sang qu'elle faisait circuler dans les veines de ces pâles fantômes leur donnait l'apparence de vie, et attirait la foule, qui ne court que là où quelque chose palpite: s'imagine-t-elle par hasard que ce sombre regard, cette voix rauque et profonde, cette pâleur maladive, ce front chargé de toutes les mélancolies de notre âge, ce jeu nerveux, cette rage froide, ce débit dégagé de mélopée, où la césure, la rime et la période poétique se font à peine sentir, ne soient pas de bel et bon drame, et nullement de la tragédie comme on l'entendait dans le grand siècle $[\ldots]^{16}$.

Les adversaires du romantisme englobent d'ailleurs dans leurs critiques de ce mouvement, l'actrice Rachel. En 1842 un auteur qui ne signe que par des initiales L. $\mathrm{P}^{\star * *}$ livre Quelques observations sur l'Art théâtral: la Comédie-Française et Melle Rachel. Ce texte est un violent réquisitoire contre l'un des principes du romantisme qui «s'autorise sottement de cette proposition, qu'on naît poète, peintre, comédien, etc. » et se transforme en pamphlet teinté d'antisémitisme contre $M^{\text {lle }}$ Rachel et son «instinct théâtral» :

C'est depuis cette seconde représentation d'Andromaque que mademoiselle Rachel, prônée par les juifs et assurée par les claqueurs stipendiés,

13. Alfred de Musset, "Rachel», La Revue des deux-mondes, $1^{\mathrm{er}}$ novembre 1838 .

14. Titre du livre de Martine Lavaud, Théophile Gautier, militant du romantisme, Paris, Champion, 2001.

15. Sylvie Vieilledent, «Gautier critique de Rachel».

16. Théophile Gautier cité par Sylvie Vieilledent, "Gautier critique de Rachel ». 
fut proclamée actrice modèle, devant faire école, et successivement, dans la même année, grande, célèbre et sublime actrice!

Mais ces critiques, si l'on ne peut les nier, sont loin d'être majoritaires. La "gloire [de Rachel] contribua fortement, comme celle de Mendelssohn, d'Halévy, de Meyerbeer et de Heine, à la construction du discours romantique sur le "potentiel créateur des juifs" " ${ }^{17}$. Mais cette "vraie fleur biblique épanouie, on ne sait comment, sur ce fumier» selon l'expression de Théophile Gautier, masque une autre approche des romantiques sur le peuple juif: le «fumier» dont parle Gautier, c'est la famille Félix, des juifs étrangers ${ }^{18}$ misérables et forcément «cupides» qui alimentent depuis toujours les pires préjugés: derrière la louange du potentiel créateur des juifs se cachent les stéréotypes antijudaïques ${ }^{19}$ en vogue depuis la fin de l'Empire Romain et surtout depuis le Moyen Âge.

Un article, en 1846, des Archives Israélites ${ }^{20}$ fustige avec humour cette prédominance des juifs de convention dans le théâtre français:

De grâce, messieurs les dramaturges, cherchez ailleurs de la couleur locale (ils appellent cela de la couleur locale) et laissez pour un instant reposer le judaïsme et les prétendues études de mœurs qu'il vous inspire. On nous a si longtemps massacrés pour de bon qu'avant de continuer à nous mettre en "pièces», il serait humain de nous laisser reposer un peu ${ }^{21}$.

Victor Hugo avait été attaqué par les Archives israélites en 1843 lors de la sortie des Burgraves, pièce dans laquelle un personnage accuse les juifs d'avoir égorgé un enfant pour leur sabbat. Blessé par cette attaque, Victor Hugo avait écrit au directeur des Archives israélites une longue lettre:

17. Anne Hélène Hoog, «"L’enfant du miracle": ambivalences du discours sur les origines de Rachel et de son génie», Rachel, une vie pour le théâtre 1821-1858, Paris, Musée d’art et d'histoire du Judaïsme - Adam Biro, 2004.

18. Jacques et Esther Félix, juifs apatrides d'origine allemande et polonaise, colporteurs, acteurs et chanteurs ambulants sont misérables et vont très vite considérer leurs filles aînées (ils ont 6 enfants) Rachel et Sarah, qui chantent dans les rues, comme des sources de revenus.

19. «Il faut bien se garder de confondre deux choses très différentes: l'antisémitisme, idéologie laïque du XIX ${ }^{\mathrm{e}}$ siècle, mais qui n'apparaît sous ce nom qu'après 1870 , et la haine du juif, d'origine religieuse, inspirée par l'hostilité réciproque de deux fois antagonistes.» Hannah Arendt, Sur l'Antisémitisme, Paris, Seuil (Points Essais), 1973. Même si l'antisémitisme moderne (qui va ajouter les préjugés de race et les préjugés politiques aux préjugés religieux) s'inspire des nombreux poncifs et superstitions populaires médiévales.

20. Journal crée par Samuel Cahen en 1840.

21. Cité par Béatrice Philippe, Être juif dans la société française du Moyen Âge à nos jours, Paris, Éditions Montalba, 1979. 
Vous m'avez mal compris, Monsieur, et je le regrette vivement, car ce serait un vrai chagrin pour moi d'avoir affligé un homme comme vous, plein de mérite, de savoir et de caractère. Le poëte dramatique est historien et n'est pas plus maître de refaire l'histoire que l'humanité. Or, le treizième siècle est une époque crépusculaire; il y a là d'épaisses ténèbres, peu de lumière, des violences, des crimes, des superstitions sans nombre, beaucoup de barbarie partout. Les juifs étaient barbares, les chrétiens l'étaient aussi; les chrétiens étaient les oppresseurs, les juifs étaient les opprimés; les juifs réagissaient. Que voulez-vous, Monsieur? C'est la loi de tout ressort comprimé et de tout peuple opprimé. Les juifs se vengeaient donc dans l'ombre, comme je vous le disais dans ma lettre; fable ou histoire, la légende du petit enfant Saint-Werner le prouve. Maintenant, on en croyait plus qu'il n'y en avait; la rumeur populaire grossissait les faits; la haine inventait et calomniait, ce qu'elle fait toujours; cela est possible, cela même est certain; mais qu'y faire? Il faut bien peindre les époques ressemblantes; elles ont été superstitieuses, crédules, ignorantes, barbares; il faut suivre leurs superstitions, leur crédulité, leur ignorance, leur barbarie; le poëte n'y peut mais, il se contente de dire: c'est le treizième siècle, et l'avis doit suffire. Cela veut-il dire qu'au temps où nous vivons, les juifs égorgent et mangent les petits enfants? Eh! Monsieur, au temps où nous vivons, les juifs comme vous sont pleins de science et de lumière, et les chrétiens comme moi sont pleins d'estime et de considération pour les juifs comme vous.

Et Hugo termine sa plaidoirie par «Amnistiez donc Les Burgraves, et permettez-moi, Monsieur, de vous serrer la main ${ }^{22}$.

Cet argument n'aurait pu servir ni à Alfred de Vigny pour La Maréchale d'Ancre qui sera jouée en 1831 au théâtre Royal de l'Odéon ni à George Sand pour Les Mississippiens car, eux, créaient un personnage de juif que rien, dans la situation historique, ne justifiait. Dans La Maréchale d'Ancre, le drame historique d'Alfred de Vigny, qui met en scène le Prince de Condé, Borgia, Concini et d'autres personnages politiques, un seul est entièrement inventé par l'auteur, le juif Samuel Montalto qui est ainsi décrit dans les didascalies: "Le juif Samuel Montalto - riche et avare, humble et faux - juif de cour. Pas trop sale au dehors, beaucoup en dessous. Beau chapeau et cheveux gras» et à l'acte II «Le laboratoire de Samuel-le-juif est assis à sa table et compte les pièces d'or». Ce juif est un avatar du Shylock du Marchand de Venise de Shakespeare que Vigny vient de traduire et qui, d'ailleurs, dans cette adaptation très libre a perdu «jusqu'aux vertus que lui accordait Shakespeare. Il accumule sur sa personne tous les poncifs

22. Victor Hugo, «lettre du 11 juin 1843», Correspondance, t. I, 1896 (http://fr.wikisource. org/wiki/Correspondance_de_Victor_Hugo_-_Tome_I_-_29). 
médiévaux ${ }^{23}$. Si l'abject Samuel Montalto de Vigny est un personnage secondaire, il n'en a pas moins une grande importance dans la pièce.

Moyennant de fortes sommes d'argent, Samuel est simultanément au service de plusieurs partis antagonistes [...]. Il trahit tour à tour Borgia et Concini, et n'embrasse le parti du Roi que sous la menace de mort ${ }^{24}$.

Grâce à son extrême habileté, il a réussi à devenir «juif de cour» et, à ce titre, il a tendance à renier ses origines. "Ne me nommez pas Samuel ici», dit-il à l'un des gentilshommes de la Cour. "J'ai pris un nom de Chrétien; je m'appelle Montalto à Paris» ${ }^{25}$.

Dans Les Mississippiens (1840), proverbe en trois actes de George Sand, le personnage de Samuel Bourset dont le patronyme transparent fait référence à la Bourse, est devenu le comte de Puymonfort masquant lui aussi sa véritable origine; d'après l'un des personnages de la pièce «Samuel Bourset s'est "décrassé" depuis son mariage», ce qui ne l'empêchera pas de vendre sa fille Louise au plus offrant:

Ô mon père! Ô ma mère! Je me plaisais encore à douter de mon isolement en ce monde; à présent, je ne le puis plus... Haïe, méprisée, livrée comme une vile marchandise dont on trafique... Oh mieux vaudrait être morte!

s'écrie la jeune fille qui vient de comprendre le marché qu'a conclu son père. Et la référence à Shylock ne se fait pas attendre: Georges Freemann (il est le héros, il porte le nom «d'homme libre » par opposition à Bourset inféodé à l'argent) s'exclame:

Ô corruption, ô âme dépravée! Femme sans entrailles et sans cour! Et toi, Samuel! Shylock moderne, il ne te reste plus qu'à tuer tes victimes, pour vendre plus aisément leur chair et leur sang!

La femme de Samuel Bourset devant le sort réservé à sa fille se réveille de seize années de mariage forcé - c'est une aristocrate ruinée qui a dû épouser un juif pour sauver sa famille:

Je vous ai aidé jusqu'ici dans vos projets de fortune - dit-elle à son mari -; j'ai partagé vos richesses et votre enivrement. J’ai même été vaine, ambitieuse, et $\mathrm{j}$ 'en rougis; mais vous aviez ennobli ce vice à mes yeux en me faisant

23. Luce A. Klein, Portrait de la Juive dans la littérature française, Paris, Nizet, 1970.

24. Joseph Sungolowsky, «Les juifs et le judaïsme dans l'œuvre d'Alfred de Vigny», PMLA, vol. $80, \mathrm{n}^{\circ} 3$, juin 1965 .

25. Ibid. 
croire que nous accomplissions une grande œuvre, que notre luxe faisait prospérer la France, et que nous étions au nombre de ses bienfaiteurs. Si je restais votre dupe un jour de plus, je serais forcée de me regarder comme votre complice, car je sais que nous ne sommes plus que des spoliateurs [...]. Vous reprendrez tous les diamants que vous m'avez donnés; je ne veux plus rien qui me rappelle que ces misérables jouets ont ruiné plus de cent familles...

Comme dans la pièce de Vigny, le juif de George Sand est un traître et qui plus est un juif qui ruine la France: sur une toile de fond politique réelle, une Compagnie fondée à Paris par le financier écossais Law pour exploiter la Nouvelle-Orléans et qui ruinera les actionnaires, George Sand ${ }^{26}$ invente cet intermédiaire juif qui préfigure tous les banquiers véreux qui peupleront la scène française de la fin du XIX ${ }^{\mathrm{e}}$ siècle.

\section{Wagner et la création de l'antisémitisme culturel}

Wagner a donc écrit en 1842 quatre articles dans La Revue et Gazette musicale, pour saluer le talent de Fromenthal Halévy particulièrement dans l'opéra La Juive:

C'est dans La Juive que la véritable vocation d'Halévy se manifesta d'une manière irréfutable [...] en essayant de caractériser sa musique, il importait de signaler d'abord les profondeurs; c'est son point de départ, c'est de là qu'il envisage l'art musical. Je ne parle point de cette passion sensible, passagère, échauffant le sang pour s'éteindre aussitôt: je parle de cette faculté de s'émouvoir, puissante, intime et profonde, vivifiant et bouleversant le monde moral de tout temps ${ }^{27}$.

\section{Et Wagner de regretter que}

[les] jeunes compositeurs français n'aient pu trouver la force de suivre les traces de l'auteur de La Juive. Et ce qu'il y a de plus déplorable - poursuit-

26. George Sand a certainement été influencée pour cette pièce par les théories de son ami Pierre Leroux (1797-1871). Considéré comme l'introducteur en France du mot «socialisme», Leroux qui écrira comme Toussenel un ouvrage intitulé Les juifs rois de l'époque, éprouve une solide amitié pour George Sand qu'il a rencontré en 1835. De même que Charles Fourier (1772-1837), Leroux considère que «l'admission des juifs au droit de cité» a été une erreur de la Révolution française car "la nation juive» a envahi "les fonctions improductives» c'est-à-dire celles du commerce.

27. Richard Wagner, «Halévy, la Juive et la Reine de Chypre» Didier Van Moere (éd.), L'Avant Scène, $\mathrm{n}^{\circ}$ 100, juillet 1987, Fromental Halévy, La Juive. 
il -, c'est qu'ils ont eu la lâcheté de subir l'influence des compositeurs italiens à la mode. Je dis lâcheté, parce qu'en effet ce me semble une faiblesse coupable et honteuse de renoncer à ce que l'on trouve de bien dans son propre pays, pour singer les médiocrités étrangères $[\ldots]^{28}$.

Dans cette louange, Wagner considère Halévy comme un créateur français à part entière.

Quelques années plus tard en 1850, changement total de discours dans le pamphlet que Wagner écrit, Le Judaïsme dans la musique:

[...] Pour le juif, toute la civilisation et tout l'art européens sont restés choses étrangères, car il n'a pas plus participé à la formation de la première qu'au développement du deuxième et il est le plus souvent resté un spectateur froid, sinon hostile. Le juif ne peut donc, dans cette langue faire œuvre de poète ou d'artiste: il devra se contenter d'imiter, de répéter ${ }^{29}$.

Oublié «le génie créateur» de Meyerbeer et d’Halévy, reniées ses analyses sur la profondeur de La Juive que les compositeurs français se devaient de prendre comme modèle. Wagner est catégorique: «Il n'existe pas d'art juif, par conséquent point non plus de vie créatrice d'art». Cet axiome et son corollaire «le goût artistique du public a été livré aux mains mercantiles des juifs » vont connaître un grand succès: d'Édouard Drumont à Lucien Rebatet, d'Octave Mirbeau à Romain Rolland, de Léon Daudet à Lucien Dubech ${ }^{30}$, le mythe de la culture «enjuivée» aux mains de «mercanti » et non de "créateurs » a traversé toutes les époques jusqu'à la solution finale déjà préconisée par Wagner: le dernier mot de son texte est «anéantissement».

En Russie, l'assassinat du tsar Alexandre II est suivi de terribles pogromes qui jettent de 1881 à 1883 de nombreux juifs sur les routes de l'exil. La gare de l'Est voit arriver des milliers de juifs pauvres ne parlant pas un mot de français. Victor Hugo publie dans Le Rappel du 19 juin 1882 un article intitulé «Les juifs - La Russie»:

[...] Le christianisme martyrise le judaïsme; trente villes (vingt-sept, selon d'autres) sont en ce moment en proie au pillage et à l'extermination; ce qui se passe en Russie fait horreur; là un crime immense se commet [...].

28. Ibid.

29. Richard Wagner, Le Judaïsme dans la musique, Paris, Société d'Édition Muller et $\mathrm{C}^{\mathrm{ie}}$, nd.

30. Pour l'antisémitisme de tous ces auteurs, voir Chantal Meyer-Plantureux, Les Enfants de Shylock ou l'antisémitisme sur scène, Bruxelles, Éditions Complexe, 2005. 
Plus loin Hugo rappelle des massacres comparables: «l'Inquisition, la SaintBarthélemy...». À quatre-vingts ans, l'auteur accepte la présidence d'un comité de secours pour les victimes des pogroms. Et il publie sa pièce Torquemada, une façon de protester contre le fanatisme religieux: la scène où le Grand Rabbin vient implorer le Roi de sauver le peuple juif est, dans le contexte politique de cette fin du XIX ${ }^{e}$ siècle, particulièrement bouleversante. Et l'indifférence à ces massacres révolte Hugo qui, dans plusieurs vers de sa pièce, laisse éclater cette indignation:

Le Roi: Bien. Que demandent-ils?

Le Marquis: Qu'on les laisse tranquilles.

Le Roi: C'est beaucoup. Je ne puis laisser tranquillement

Des hommes être juifs. [...]

Le Marquis: Les juifs, Sire, industrieux, nombreux,

Demandent, prosternés, que le roi les tolère

En Espagne, et les voie à ses pieds sans colère,

Et révoque l'édit qui les exile.

Le Roi : Alors,

Que veulent-ils?

Le Marquis: Mourir où leurs pères sont morts, Rester dans leur pays, Sire, et je vous présente Leur rançon. Prenez-la.

La pièce sera republiée en 1933 et le journaliste des Nouvelles Littéraires montre la résonance de ce texte avec les évènements qui se déroulent alors en Allemagne ${ }^{31}$.

La même année 1882, le krach de la banque catholique, l'Union Générale, créée quatre ans plus tôt "pour lutter contre les banques juive et protestante», sera attribué à tort aux Rothschild; les dirigeants catholiques auront beau être condamnés pour pratiques frauduleuses, la haine à l'égard des juifs se propage: la Croix devient un quotidien qui se proclame «le journal le plus anti-juif de France», les feuilles antisémites fleurissent. Octave Mirbeau devient le rédacteur en chef des Grimaces et annonce tout de suite la couleur: "Les Grimaces seront très antirépublicaines et très antijuives. Cela va ronfler...» ${ }^{32}$. Mirbeau, avant même La France juive, mêle les préjugés antisémites des socialistes sur le rapport des juifs à l'argent, aux poncifs wagnériens sur leur absence de créativité.

31. Pierre Paraf, Nouvelles littéraires, 28 octobre 1933.

32. Lettre citée par Jean-François Nivet et Pierre Michel, Octave Mirbeau, l'imprécateur au cour fidèle, Paris, Librairie Séguier, 1990. 
L'industrie théâtrale si facile à des développements d'affaires devait tout naturellement séduire les juifs nécessiteux et dévoyés qui jusqu'au glorieux Scribe [Scribe est considéré comme un auteur dramatique juif; le succès populaire est si suspect qu'il ne peut s'agir que d'un écrivain juif], n'avait fait «du théâtre» qu'en vendant des lorgnettes dans la salle et qu'en prêtant, à gros intérêts, des parures de diamants et de perles aux actrices mal entretenues. Les juifs n'aiment pas à pratiquer l'art pour l'art, et trouvent que la poésie n'est bonne que pour les chrétiens qui se nourrissent de rêves, et non de belles pièces d'or, les imbéciles! [...] Enfin M. Scribe vint, et le premier en Israël, sut mettre la main sur les théâtres et les exploiter, âprement, comme il convenait. Il fut le grand initiateur qui découvrit un débouché nouveau aux appétits des juifs, une branche de commerce non encore explorée par eux et qui devait porter les fruits pourris que l'on sait [...].

Les juifs, qui se rendent parfaitement compte de la répugnance qu'ils inspirent, dissimulent volontiers leur tare originelle, et facilement entrent dans des métiers et des professions d'apparence noble, où ils espèrent cacher à jamais les tendances et les fatalités de leur race. Ils se sont rués sur le théâtre, poussés par leur désir du gain et aussi par cette idée qui leur fait considérer les professions qui ne dérivent ni de la Bourse, ni de la Banque, comme une sorte d'affranchissement, d'anoblissement. Ils ont des façons de regards et des gestes qui semblent vous dire: "Mais je ne suis pas un juif, je suis un auteur dramatique.» Ce qui d'ailleurs ne les empêche pas, en rimant des chansons d'opérettes ou en charpentant des drames émouvants, d'occuper dans des Sociétés de crédit, des situations clandestines, à l'ombre desquelles ils suivent, d'un œil bordé de rouge et d'un doigt crochu, des opérations véreuses et lucratives.

Le théâtre aujourd'hui leur appartient à peu près exclusivement... [...]

Et le public qui, dans la journée est allé jeter son argent aux guichets des juifs, brasseurs d'affaires, vient encore vider sa bourse, le soir aux bureaux des juifs, triporteurs de basse littérature. Là-bas on lui a pris sa fortune, ses économies; ici on lui prend son esprit, son goût, son respect On le ruine dans la journée, et la nuit, on l'avilit et on l'énerve. Étouffé dans les serres grippantes du monstre juif, il est devenu ce public hébété, dont le cœur vidé et les mains inertes ne battent plus aux œuvres généreuses et dont les sens seuls ne s'éveillent qu'aux puanteurs de l'ordure. [...] En voyant les juifs essayer de sortir de leur métier traditionnel, et de s'élever des boutiques d'usurier jusque dans les régions sereines de l'art et de la pensée, instinctivement on serait porté à applaudir à cette aspiration inespérée. Malheureusement ce ne sont pas les juifs qui se sont haussés jusqu'à la littérature; c'est elle qu'ils ont abaissée jusqu'au niveau de leurs comptoirs. Ils l'ont mise dans la même balance où ils pèsent leurs 
pièces d'or, et ils la traînent dans les mêmes trafics et les mêmes spéculations sordides ${ }^{33}$.

Cette longue citation d'Octave Mirbeau ${ }^{34}$ permet de comprendre que cette notion de "théâtre enjuivé», qui a remporté le succès que l'on sait sous l'Occupation ${ }^{35}$, n'est pas une invention des collaborateurs ou des nazis mais un concept qui prend ses racines à la fin du XIX ${ }^{\mathrm{e}}$ siècle : ce n'est pas une parenthèse honteuse de la Seconde Guerre mondiale mais la continuité d'une idée qui a irrigué la société française durant presque un siècle et cela explique la facilité avec laquelle cette conception s'est imposée sans contestation réelle durant ses années noires.

C'est le livre d'Édouard Drumont (1844-1923) La France juive, paru en 1886 , qui va diffuser très largement ${ }^{36}$ les idées déjà développées par Octave Mirbeau:

Le théâtre lui-même a pris une importance anormale, presque monstrueuse qui s'explique par ce seul fait que la plupart des artistes en renom sont juifs. Le métier de comédien devait tenter les juifs; il rapporte beaucoup, en effet, il satisfait une certaine vanité subalterne et il ne demande aucune faculté géniale; ils se sont rués sur cette carrière avec une véritable fureur.

Drumont a expliqué quelques pages avant par «la névrose juive » ce besoin de «publicité » qui trouve son exutoire au théâtre et à l'opéra et par «l'hystérie» cette frénésie sur scène ${ }^{37}$. S'ensuit une longue liste de noms censés démontrer cette maxime: «Tous les théâtres de Paris sont aux mains des juifs.» Et

il s'est produit - affirme-t-il - ce qui s'est produit partout où les juifs apparaissent; ils sèment le désordre et la ruine sur leur passage. L'Opéra qui, depuis l'abbé Perrin (1671) et Lulli, avait toujours été à peu près [bien], a cessé brusquement de marcher dès que les juifs s'en sont mêlés.

33. Octave Mirbeau, «Le théâtre juif », Les Grimaces, 3 novembre 1883.

34. Octave Mirbeau se repentira d'avoir écrit cette chronique (et d'autres) sur les juifs et deviendra un ardent dreyfusard mettant sa notoriété et son argent au service de la défense de Dreyfus et de Zola.

35. L'exposition Le juif et la France inaugurée à Paris au Palais Berlitz le 6 septembre 1941 reprendra dans les espaces consacrés au théâtre et au cinéma tous les thèmes développés par Mirbeau dans ce pamphlet.

36. Véritable best-seller avec ses 114 éditions en un an et son édition populaire abrégée.

37. «Névrose», "goût de la publicité» et «hystérie», trois des épithètes qui seront accolés à Sarah Bernhardt (et à d'autres comme l'acteur De Max). 
Si le juif est reconnaissable à son patronyme, il l'est aussi par son physique $^{38}$ :

Les principaux signes auxquels on peut reconnaitre le juif restent donc: ce fameux nez recourbé, les yeux clignotants, les dents serrées, les oreilles saillantes, les ongles carrés au lieu d'être arrondis en amande, etc. ${ }^{39}$.

Le message est simple, répétitif, écrit dans une langue rudimentaire à la limite du langage parlé pour être compris de tous. Ces arguments se diffuseront bientôt quotidiennement dans La libre Parole puis dans La libre Parole illustrée dans lequel «l'image doit compléter l'œuvre de la plume». Le théâtre et la caricature y tiendront une place particulièrement importante. C'est Jean Drault, lui-même auteur dramatique et dessinateur qui sera responsable de ses deux rubriques. Durant près d'un demi-siècle - il reprendra du service pendant la Seconde Guerre mondiale - Drault traquera les auteurs et les acteurs juifs, défendra les pièces antisémites, provoquera des cabales.

\section{Sarah Bernhardt, star et cible de l'antisémitisme moderne}

Il est donc intéressant de comparer le traitement qu'a subi Sarah Bernhardt à la fin du siècle, c'est-à-dire après les Grimaces de Mirbeau et La France juive de Drumont, à celui qu'a connu Rachel au début du siècle pour déceler l'évolution de l'antisémitisme au cours du XIX siècle. Comme l'écrit très justement Anne-Hélène Hoog dans le catalogue de l'exposition que le musée d'Art et d'Histoire du Judaïsme a consacré à Rachel:

Les premiers biographes et critiques de Rachel mentionnent sa judéité sans s'y attarder. Elle était de notoriété publique, ne jouait qu'un rôle secondaire et offrait davantage un certificat d'aptitude au tragique qu'un obstacle à sa carrière.

Mais l'historienne constate que «les biographies publiées dans les décennies qui suivirent la mort de Rachel [c'est-à-dire contemporaines de Sarah Bernhardt] sont d'un esprit différent. La vision de l'artiste se figea, la légende prit son essor et les clichés antisémites eurent libre cours.» Les mêmes qualificatifs - instable, excessive, âpre au gain et manipulatrice -

38. Cette description correspond à l'homme juif.

39. Édouard Drumont, La France juive, Paris, Marpon et Flammarion éditeurs, nd. 
désigneront Rachel et Sarah Bernhardt en cette fin du XIX ${ }^{\mathrm{e}}$ siècle bouleversée par l'Affaire Dreyfus. Rachel est morte et ces attaques posthumes ne peuvent plus l'atteindre mais Sarah Bernhardt, qui n'a pas caché son soutien à Zola et à Dreyfus, va devenir la cible privilégiée des antisémites : l'affaire Marie Colombier du nom de l'actrice qui écrivit un pamphlet antisémite Sarah Barnum en est l'exemple le plus symbolique ${ }^{40}$. Le livre fait un tableau de l'actrice à grands traits antisémites : la description physique est déjà largement tendancieuse ${ }^{41}$ :

grande et mince, maigre à en être ridicule, la jeune comédienne avait une tête d'un caractère étrange. Les traits étaient corrects, d'un dessin pur, dont les lignes rappelaient, mais en l'adoucissant encore et en l'affinant, le masque juif de la mère. Pourtant on n'eût pas pu davantage dire que ce visage fût joli, qu'affirmer sa laideur. Quelque chose de fuyant, de non-formé le noyait. [...] En vraie fille d'Israël, la Barnum était sale [...];

suivent les accusations de cupidité et de décadence des mœurs:

Mademoiselle Barnum n'avait de sa race que l'âpreté au gain, sans posséder l'habileté de celle-ci à le faire naître [...].

Les amis ou amants de Sarah Barnum ont « des regards luisants, chauds de désir; [leur] lèvre inférieure exsangue et pendante tremblotait, baveuse » et elle propose cette définition de l'amitié chez les juifs: «dans la bouche du juif, amis voulait dire clients. » Mirbeau dont on a vu les premiers pamphlets antisémites, se révolte contre ce qu'il appelle « un crime de librairie»:

À la lecture de ces pages, il nous monte aux lèvres un tel dégoût, une telle colère vous vient contre la mauvaise et lâche action de ces deux misérables ${ }^{42}$, qu'instinctivement vous êtes pris d'une grande pitié pour cette femme si odieusement roulée dans de telles fanges, et vous éprouvez, vous qui ne l'aimez pas, vous qui auriez peut-être de la haine et du mépris pour elle, le besoin de la défendre ${ }^{43} \ldots$

40. Marie Colombier (qui avait été l'amie de Sarah Bernhardt durant de nombreuses années) poursuivie pour outrages aux bonnes mœurs, a comparu le 26 mai 1884 devant la Cour d'assises de la Seine, et a été condamnée à trois mois de prison et 1000 francs d'amende. Le volume a été saisi par ordre de la Cour et a été remis plus tard en vente avec la suppression des passages incriminés.

41. Voir la photographie très connue de Sarah Bernhardt par Nadar.

42. Octave Mirbeau parle de l'auteur Marie Colombier et de son préfacier Paul Bonnetain, journaliste et écrivain maritime et colonial.

43. Les Grimaces, 15 décembre 1883. 
Mirbeau provoquera en duel Paul Bonnetain et deviendra l'ami de Sarah Bernhardt: il regrettera publiquement son antisémitisme ${ }^{44}$.

Si toutes les critiques n'atteignent pas ce niveau d'abjection, elles sont néanmoins très souvent teintées d'antisémitisme. Les caricatures qui les accompagnent sont extrêmement révélatrices de cet antisémitisme: l'identité juive de Sarah Bernhardt est toujours signalée (alors qu'elle est convertie au catholicisme):

- l'étoile de David accompagne de nombreuses caricatures (fig. 2, 3 et 5);

- le nez grossièrement recourbé symbole de la judéité (même si toutes les photos montrent un nez droit) (fig. 4);

- le rappel de l'argent (tas d'or) (fig. 5);

- le caractère "monstrueux» de l'actrice: elle est une sorcière avec un balai, elle sera croquée en Sphinge aux doigts crochus ${ }^{45}$ (surnom qu'on lui donnait; la sphinge est cruelle contrairement au sphinx égyptien bon et protecteur ${ }^{46}$ ) et en chimère. Or la sphinge, sœur de la chimère, fait partie de la même famille que les Harpyes ${ }^{47}$, déesses des tempêtes et ravisseuses (fig. 4 et 5).

Sarah Bernhardt est comparée à ces monstres «femelles» qui terrorisent et dévorent les êtres qui ont le malheur de se trouver sur leur route. Elle subit une double condamnation en tant que juive et en tant que femme libre.

\section{Juive et femme, un antisémitisme genRé}

L'antisémitisme dont a été victime Sarah Bernhardt correspond à des courants politiques spécifiques - nationaliste et socialiste - mais aussi à une montée des revendications féministes. Il faut en effet différencier les traitements dont sont l'objet les juifs et les juives sur scène. À y regarder superficiellement, les femmes juives semblent beaucoup mieux traitées que les

44. Lors d'une conférence en décembre 1898, Octave Mirbeau affirmera que «l'antisémitisme, ce n'est pas une doctrine, c'est la forme la plus hideuse du banditisme et de l'assassinat », cité par Jean-François Nivet et Pierre Michel dans Octave Mirbeau, l'imprécateur au cour fidèle, Paris, Librairie Séguier, 1990.

45. Elle a joué dans Le Sphinx d'Octave Feuillet en 1873.

46. En Égypte, monstre fabuleux formé d'un corps de lion et d'une tête de pharaon, c'est l'incarnation du roi ou du dieu Soleil, symbole de puissance et de protection. En Grèce, il se transforme en démon au visage et au buste de femme et devient cruel.

47. Ce sont des divinités grecques de la dévastation et de la vengeance divine: plus rapides que le vent, invulnérables, caquetantes, elles dévorent tout sur leur passage, ne laissant que leurs excréments. 
hommes. À elles les grands rôles de tragédiennes dans les pièces classiques, à eux les rôles comiques. Dans le répertoire de la fin du XIX ${ }^{e}$ siècle et du début du XX ${ }^{\mathrm{e}}$ siècle, l'homme juif est caricatural, grotesque et pour le rendre tout à fait ridicule, il est affublé d'un nez postiche et d'une perruque rousse. C'est en général un banquier cupide, vieux, gras et libidineux ${ }^{48}$. La femme, elle, est belle et sensuelle: elle a un charme «oriental» ce qui fait dire à Maurice Bloch ${ }^{49}$ qu'il s'agit du triomphe de la femme juive:

Le mépris [que l'on a pour l'homme juif] fait place à l'estime, la haine se change en sympathie et la race maudite devient la race aimable entre toutes, digne d'être aimée et bien mieux encore! digne d'être admirée: car elle a toutes les qualités et les vertus ${ }^{50}$.

Or si la femme n'est pas ridiculisée physiquement ${ }^{51}$, ses aspirations, elles, le sont: l'un des principaux défauts de la femme juive est d'être une «cérébrale ${ }^{52}$, libérée ${ }^{53}$, et par là même un danger pour la famille française. Sarah Bernhardt revendique cette liberté:

Mes goûts un peu fantastiques, ma maigreur, ma pâleur, ma façon toute personnelle de m'habiller, mon mépris de la Mode, mon j'm'enfichisme de toutes choses, faisaient de moi un être à part ${ }^{54}$.

Elle n'acceptait aucune limitation à sa liberté, mais faisant carrière comme un homme, elle refusait de n'être qu'une actrice, et, bravant parfois les moqueries, se livrait à la peinture, à la sculpture, à la littérature, révoquant ainsi l'ordre intellectuel de cette époque... Le scandale était donc que cette femme soit libre ${ }^{55} \ldots$

48. Pour plus de précisions sur la représentation des juifs sur scène, voir Les Enfants de Shylock.

49. Conférence sur «La Femme juive au théâtre» devant la Société des Études juives, 1901 (fonds de la BDIC).

50. Maurice Bloch, Conférence sur «La Femme juive au théâtre».

51. Néanmoins Sarah Bernhardt, on l'a vu avec les caricatures, n'échappe pas à la déformation de ses traits.

52. Cette «cérébralité juive» dont parle Jouvet à propos de Judith de Giraudoux, Cahiers Jean Giraudoux, 1980, n 9, Correspondance entre Jean Giraudoux et Louis Jouvet.

53. «Elle réclame pour la femme le droit de disposer de sa personne, et elle veut agir selon ce droit » écrit Maurice Donnay de son «héroïne» juive, Judith, dans la préface à sa pièce antisémite Le Retour de Jérusalem: sous la plume de Donnay, ce n'est évidemment pas une qualité. Voir Les Enfants de Shylock.

54. Sarah Bernhardt, Ma Double Vie: mémoires, Paris, Fasquelle, 1907.

55. Claudine Herrmann, «Présentation » à Sarah Bernhardt Ma Double vie: mémoires [1907], Paris, Éditions des Femmes 2007. 
Ses nombreux rôles de travestis ${ }^{56}$

On m’a souvent demandé pourquoi j'aime tant à représenter des rôles d'hommes et en particulier pourquoi j'ai préféré celui d'Hamlet à celui d'Ophélie. En réalité, je ne préfère pas les rôles d'hommes mais les cerveaux d'hommes et parmi tous les caractères, celui d'Hamlet m'a tenté entre tous parce qu'il est le plus original, le plus subtil, le plus torturé et cependant le plus simple pour l'unité de son rêve...

ont achevé de rendre son personnage choquant pour la morale de l'époque: si l'actrice est adulée, la femme et la juive mêlées suscitent toutes les critiques. L'antisémitisme et l'antiféminisme se rejoignent dans ces critiques faites à Sarah Bernhardt. Elle menace l'ordre patriarcal de la société en revendiquant ouvertement des droits en tant que femme et actrice (celui de choisir des rôles masculins, de s'habiller en homme) et en tant que juive (son soutien à la cause dreyfusarde). Sarah Bernhardt n'est plus l'actrice singulière au destin unique comme Rachel mais celle qui embrasse les luttes de son temps. Symbole d'un monde qui change, elle devient naturellement la cible des critiques antisémites et antiféministes.

Chantal Meyer-Plantureux

56. On pourrait ajouter les allusions à l'homosexualité. Ses rôles d'homme et son amitié avec Louise Abbéma (cf. cire de Louise Abbéma Les Mains jointes de Sarah et Louise), homosexuelle reconnue, lui valurent certaines plaisanteries. 


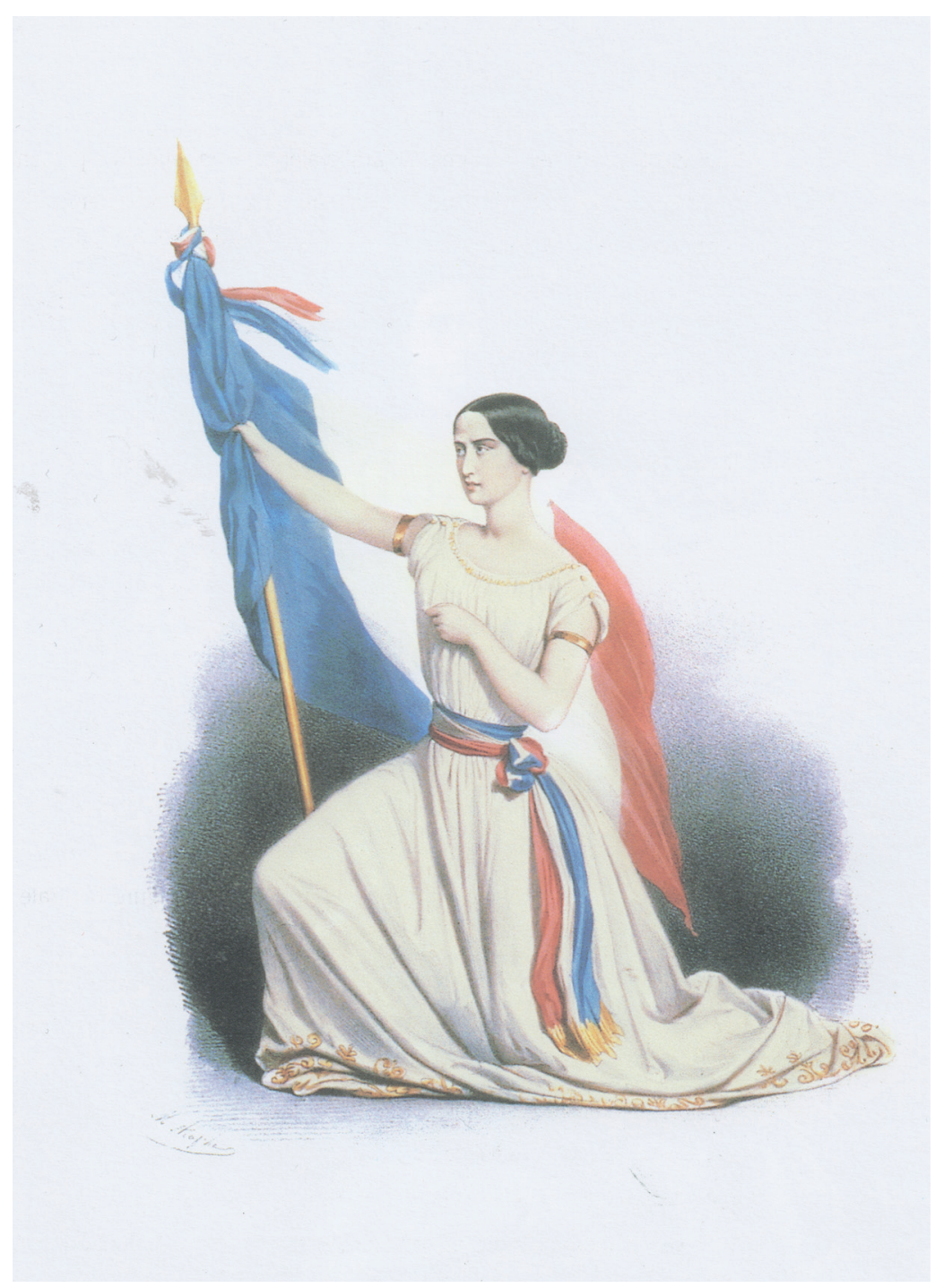

Fig. 1: Mademoiselle Rachel déclamant La Marseillaise (1848) par Marie-Alexandre Alophe dit Menut Alophe (1812-1883) 


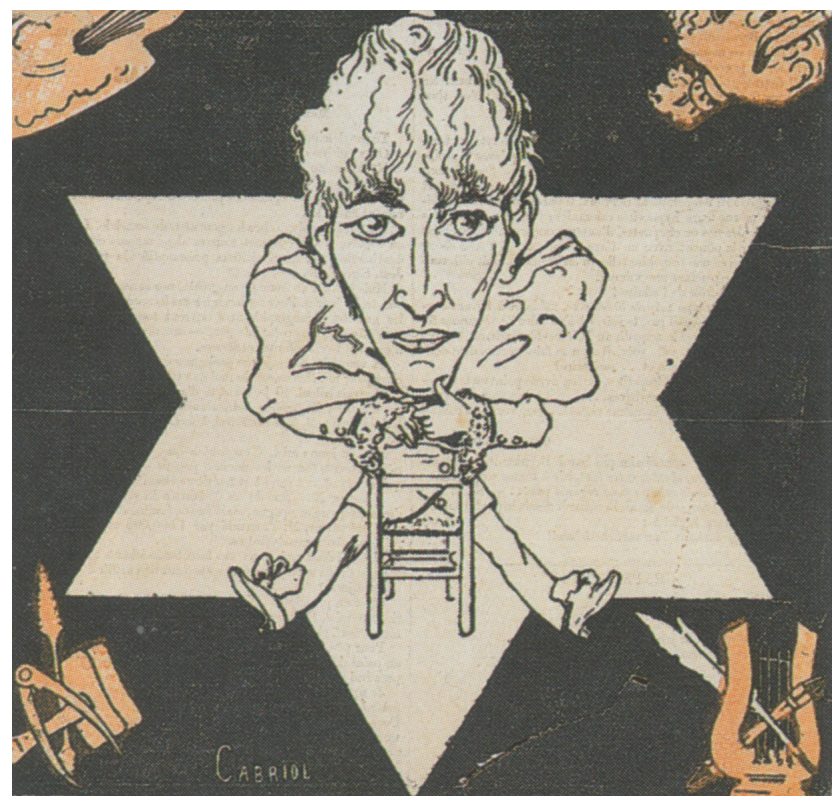

Fig. 2 : L'hydropathe Sarah Bernhardt par Cabriol (pseudonyme de Georges Lorin) (1850-1927). Cette caricature illustre un article de Georges Lorin intitulé "Monsieur Sarah Bernhardt" dans L'Hydropathe du 5 avril 1879 


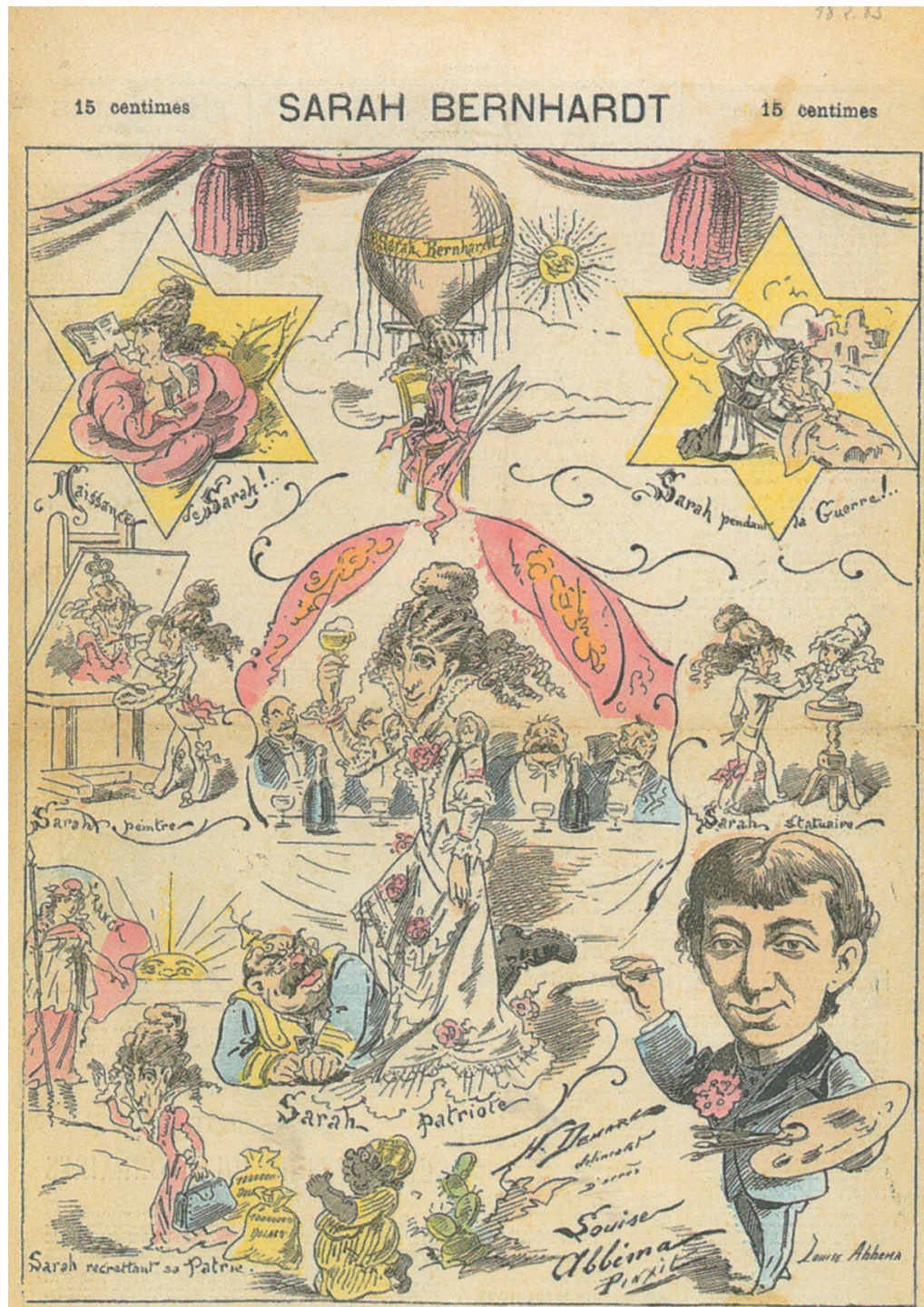

QUELQUES CHAPITRES DE SA VIE (A suivre)

Fig. 3: Sarah Bernhardt, quelques chapitres de sa vie par Henri Demare (1846-1888) 


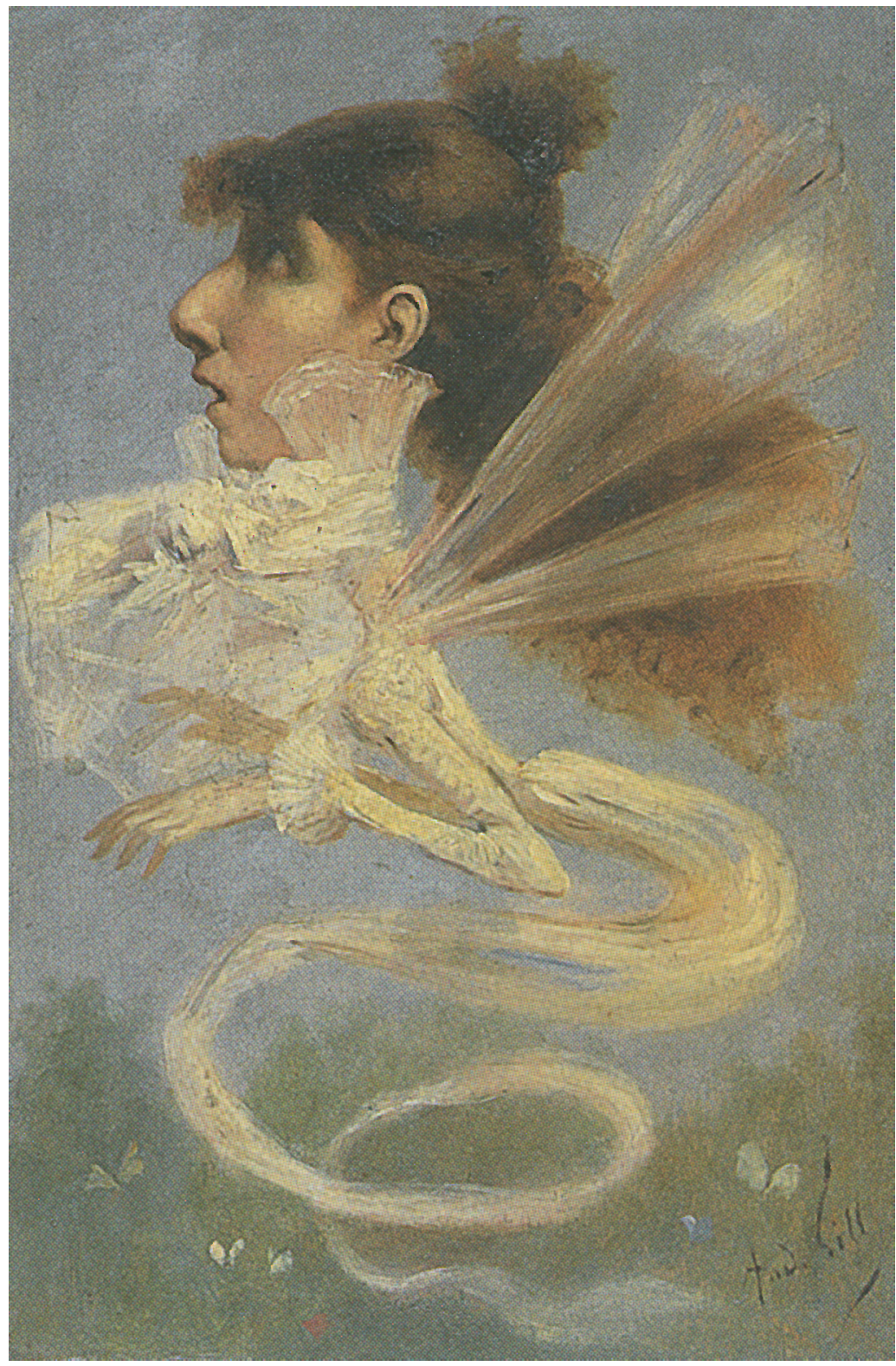

Fig. 4: Sarah Bernhardt en chimère par André Gill (1840-1885) 


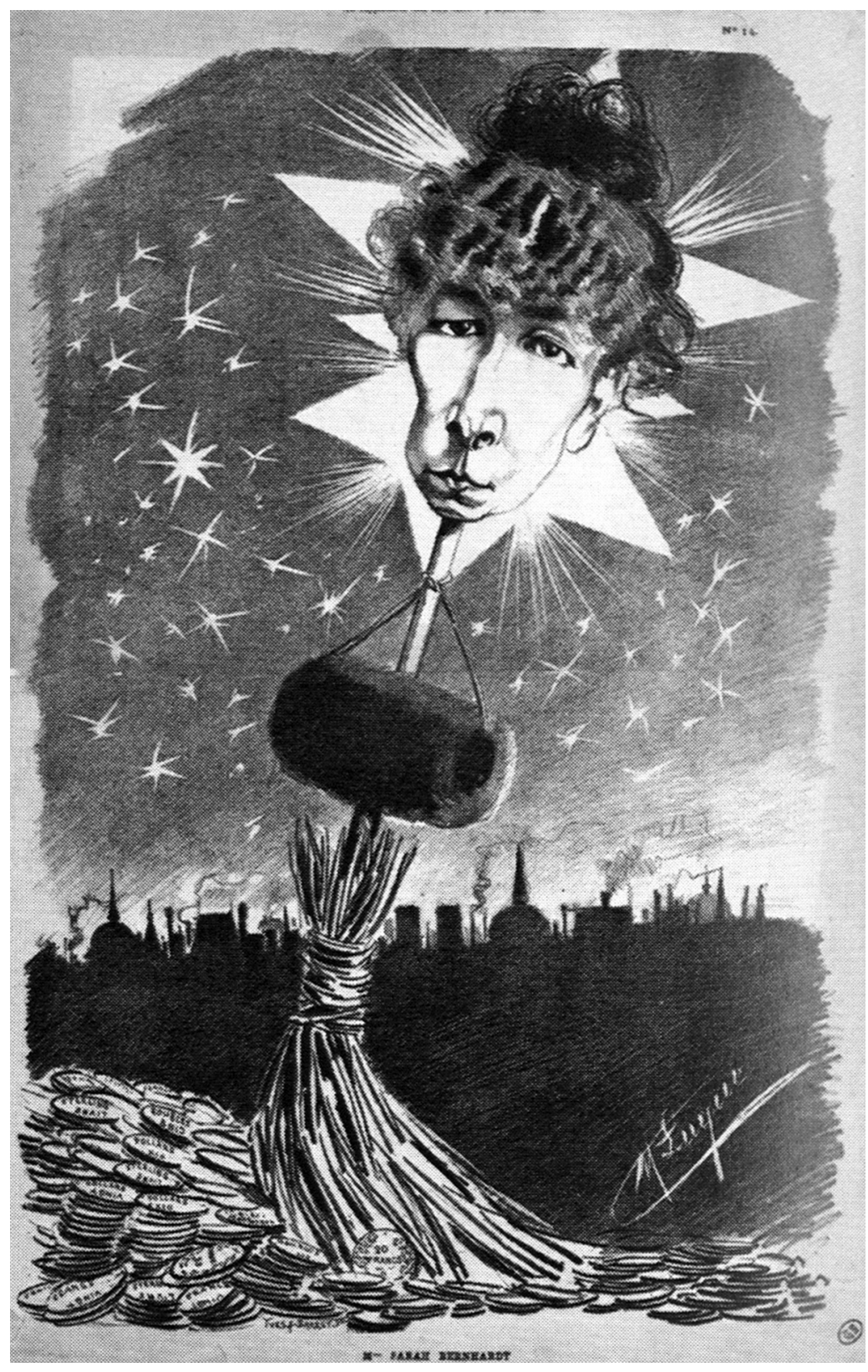

Fig. 5: Madame Sarah Bernhardt balayant la monnaie par Manuel Luque dit Luque (1854-1919) 